\title{
Modelling of Water-Assisted Flame Synthesis of Carbon Nanotube using Counterflow Diffusion
}

\author{
Muhammad 'Abid Ira Irawan'1, Mohd Fairus Mohd Yasin ${ }^{1, *}$, Khairul Fikri Tamrin² \\ 1 School of Mechanical Engineering, Faculty of Engineering, Universiti Teknologi Malaysia, 81310 UTM Johor Bahru, Johor Bahru, Malaysia \\ 2 Department of Mechanical and Manufacturing Engineering, Universiti Malaysia Sarawak, Kota Samarahan, Sarawak, Malaysia
}

\section{ABSTRACT}

Research on carbon nanotubes (CNTs) has been performed extensively. On top of that, water-assisted synthesis of CNT has started to emerge with captivating effect towards growth of CNT. The present study investigates a baseline inlet condition for water assisted case utilizing diffusion flame that imitates the temperature distribution and growth region of CNT without water vapor, on the basis of experimental data of non-water assisted high yield CNTs. To affirm the effect of water vapor, $35 \%$ to $70 \%$ of water vapor has been added replacing the fuel side nitrogen content. The results prove that water vapor suppresses the flame where the maximum temperature drops with increasing concentration. Consequently, this affects the length and growth region of CNTs. The region width has been reduced for about $7.4 \%$ to $18.5 \%$ with water vapor. This shows that excessive water poorly affects the growth of CNTs. On the other hand, the region has also shifted for about $0.64 \mathrm{~mm}$ to the fuel side $(5.7 \%)$ from the origin when $70 \%$ of water vapor was added. Following this, the impact of catalyst towards CNT growth is subsequently presented whereby a comparison is made between Fe and Co to synthesize CNT using flame synthesis. Based on the result, Fe possess better activation for the CNTs to grow as compared to Co. A significant difference between the predicted CNT length for Fe (147 $\mu \mathrm{m})$ and Co $(56 \mu \mathrm{m})$ is attributed to their diffusivity values.

Keywords:

Carbon nanotubes; flame synthesis; water-assisted; counterflow

Copyright @ 2020 PENERBIT AKADEMIA BARU - All rights reserved

\section{Introduction}

Carbon is undoubtedly an abundant element in nature which exists either in the form of solid, liquid or gaseous. In 1991, lijima successfully discovered carbon nanotubes (CNTs) [1]. With notable captivating CNTs' characteristics, a broad range of researches has been ongoing to explore the synthesis, growth mechanism and applications of CNT.

The growth process of CNTs is often accompanied by undesirable amount of carbonaceous materials and impurities due to chemical reagents and solvent contamination including the ones from gas precursor. This unintentionally would degrade quality as well as purity of the produced CNTs. For long, this issue has always been the challenge in attaining a long, pure and high quality CNTs. Hu et al., [2] stated that instigating weak oxidizer may help to enhance their growth. In particular, water vapor tends to be the excellent choice for this mild oxidizing agent as in situ

\footnotetext{
${ }^{*}$ Corresponding author.

E-mail address: mohdfairus@mail.fkm.utm.my (Mohd Fairus Mohd Yasin)
} 
purification of CNTs instead of the conventional post purification technique [3]. The study related to the addition of water vapor towards growing CNTs is seen to emerge since a number of preceding studies have shown positive results.

Liu et al., [3] found that an optimum water content is vital to obtain enhanced growth of CNTs. The appropriate amount of water is believed to behave as an etching agent to eliminate the amorphous carbon deposited on the catalyst surface [4]. For that reason, catalyst lifetime is prolonged which leads to a more continuous and stable growth of CNTs, which corroborates well with results of Hu et al., [2]. They observed that for the sample grown without water, the top part of the Single Wall Carbon Nanotubes (SWCNTs) is tilted instead of vertically aligned due to lack of activation of the water vapor.

Catalyst is the kernel of any catalytic growth of CNTs. They are accountable as a nucleation site for the growth process. In this catalytic supported CNT synthesis, no CNT will likely to be produced anymore once the catalyst deactivates. Transition metals such as cobalt, iron and nickel are considered to be the most potent of all $[4,5]$ since they have higher solubility and diffusion rate for carbon.

\section{Methodology}

A counterflow diffusion flame model was used to comprehend the integration of both flame and particle scale model. The Naha-Puretzky growth rate model was employed for the study. However, previous study only considered catalytic effect of cobalt, Co $[12,13]$. Therefore, the sensitivity of the model should be tested whereby a comparison on the basis of the resulting CNT length will be made between Co and iron, Fe. The difference of the input depends on their diffusivities value, $D_{s}$ and $D_{b}$. To analyze the effect of water vapor incorporation towards CNT growth, suitable condition must be first established to ensure stability of the flame before synthesizing products. The baseline model aims in maintaining the same increase in temperature between non-water and water assisted cases by appropriately adjusting the heat input. The concept of enthalpy of reaction is considered here.

Heat released certainly changes if water vapor is added to the reaction. Therefore, to maintain an equal amount of the heat released through this exothermic process as stated in Eq. (1), the mass flow rate of the new water-assisted diffusion flame need to be altered. This can be calculated by iteration method using the energy equation such in Eq. (2) in MATLAB. From Eq. (2), $\left(\rho_{m i x} A v\right)$ is the expansion of mass flow rate, $\dot{m}$ where $\rho_{\text {mix }}, A$ and $v$ are the density of gas mixture, area of nozzle and stream velocity respectively.

$\dot{Q}_{1}=\dot{Q}_{2}$

$\dot{Q}=\left(\rho_{m i x} A v\right) H$

$\left(\rho_{m i x, 1} A v_{1}\right) H_{1}=\left(\rho_{m i x, 2} A v_{2}\right) H_{2}$

$\bar{h}_{i}(T)=\bar{h}_{f, i}\left(T_{r e f}\right)+\Delta \bar{h}_{s, i}\left(T_{r e f}\right)$

Before equating both energy equation, enthalpy of non-water assisted and water assisted diffusion flame are calculated first. Equation related for the calculation is presented in Eq. 6 where $h_{i}(T)$ is the absolute enthalpy at temperature $T, \bar{h}_{f, i}\left(T_{\text {ref }}\right)$ is the enthalpy of formation at standard reference state and $\Delta \bar{h}_{s, i}\left(T_{\text {ref }}\right)$ is the sensible enthalpy change in going from $T_{\text {ref }}$ to $T$. To define 
standard reference state, this study employs standard-state temperature of $25^{\circ} \mathrm{C}$ and standard-state pressure of $1 \mathrm{~atm}$ equivalent to 101325 .

On the other hand, the effect of water vapor towards flame distribution and the growth region of CNT is also studied prior to developing a baseline model. Based on literature review, waterassisted CNT synthesis was solely studied using CVD method [8]. Owing to that, the water content applied in the CVD, $100 \mathrm{sccm}$ equivalent to $0.7 \%$ water vapor serves as a reference in establishing the baseline model.

\section{Results and Discussion}

\subsection{The Effect of Different Catalyst towards the Growth of CNT}

Figure 1 illustrates the resulting length of CNT using Co and Fe as catalysts. Based on the graph, Fe implies better CNT growth with outstanding length compared to Co. This result is supported by a previous study by Lee et al. [7] who synthesized long length and high density VA-CNTs by CCVD on Al supported Fe catalyst. They had validated that Fe results in best crystallinity CNTs [8].

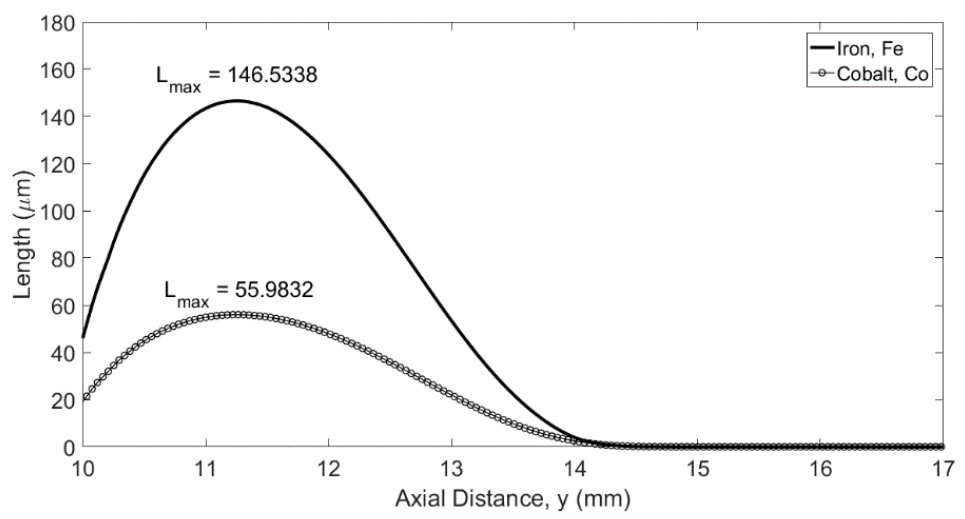

Fig. 1. Graph of CNT length for different types of catalyst

In addition, Fe has a high value of surface diffusivity. This high value of $D_{s}\left(2.09 \times 10^{-16}\right)$ exhibited by Fe nanoparticle allows more carbon atoms to be completely adsorbed at once on its surface. In comparison, Co possesses lower surface diffusivity. Nonetheless, smaller value of surface diffusivity is preferred since it contributes towards higher rate of density of $C$ atoms. Catalyst lifetime could be lengthen since accumulation of carbon atoms on the catalyst surface is not immensely saturated. This creates lower possibilities of poisonous carbonaceous layer formation that could deteriorate the metal catalyst nanoparticle (MCNP).

Another term that also affects the growth characterized by catalyst is bulk diffusivity, $D_{b}$. High value of bulk diffusivity implies eminent rate of carbon atoms diffusing into the catalyst nanoparticle. The chances of carbon atoms bounded on the surface of the catalyst to enter the particle are much greater. Essentially, both of these diffusivities work interdependently which influence the outcome of CNT growth. By assessing the values of both diffusivities of $D_{s}$ and $D_{b}$, one may notice that Co and Fe have four and six order of magnitude difference, respectively. 


\subsection{Establishment of the Baseline Model for Water-Assisted Flame Synthesis of CNT}

\subsubsection{Validation of the water-assisted baseline model}

Validation is made by comparing the simulated results having water vapor insertion with experimental data recorded by Hou and co-workers previously. Figure 2 illustrates the temperature distribution along the axial distance.

Based on the temperature profile, the water-assisted case obeys the trend of the actual measured temperature without water vapor. This shows that the baseline model with $100 \mathrm{sccm}$ of vapor content is able to predict comparable temperature profile without inclusion of the water vapor. This is highly desirable since high yield CNTs are normally produced at the said temperature range with the operated fuel content. Nevertheless, the predicted temperature profile has less fluctuation compared to the measured one that may be attributed to the exclusion of wire mesh from the model. Another study [5] noted that the discrepancy between experimental and computational data is largely caused by the uncertainty in the flow rate measurement.

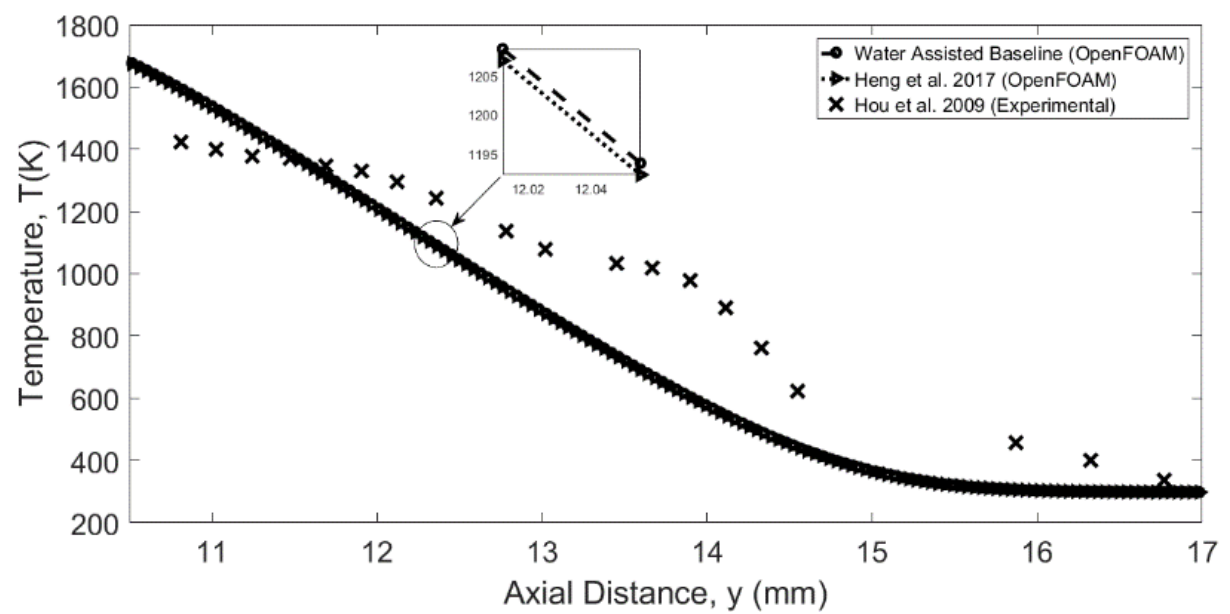

Fig. 2. Validation of simulated temperature with experimental data

The length of CNT was calculated along the axial distance from nozzles as illustrated in Figure 3. The predicted maximum CNT length $L_{\max }$ is approximately $60 \mu \mathrm{m}$. Two shaded bands of different colors have been used where grey represents the predicted growth region and black for the experimental one.

The predicted growth region is determined by considering $90 \%$ of $L_{\max }$ to obtain the bounded region of CNT growth. Based on the experimental result of Hou et al. [9], the region for CNT growth using counterflow diffusion flame lies between 12 to $13 \mathrm{~mm}$ from the oxidizer inlet whereas the resulting data from simulation shows distances ranging between 10.7 to $11.8 \mathrm{~mm}$. The deviation is roughly $1.25 \mathrm{~mm}$ from the center of both bounded regions, and is considered acceptable for the purpose of model estimation. 


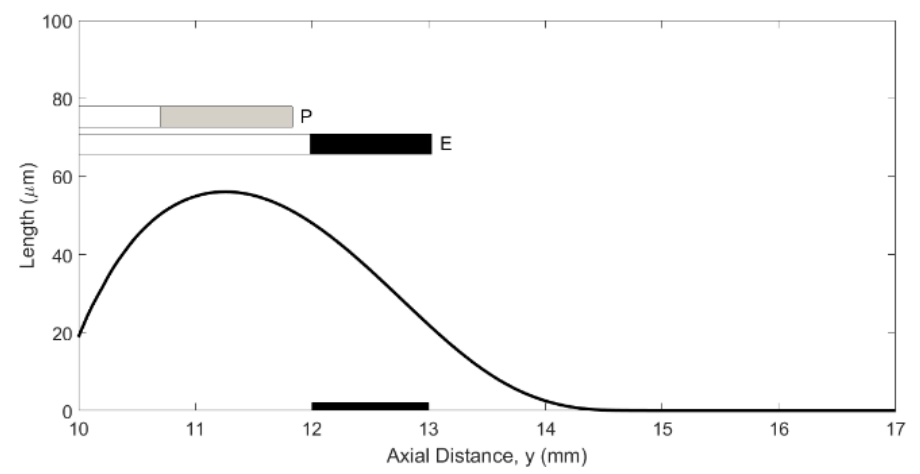

Fig. 3. Growth region of the predicted (P) and experimental (E) CNT length

\subsection{The Effect of Water Vapor Concentration towards CNT Growth and Flame Structure}

The growth region of the baseline model with water vapor content of $100 \mathrm{sccm}$ lies within a similar range as the non-water assisted flame synthesis. As the water vapor concentration changes from $35 \%$ to $70 \%$ the nitrogen dilution reduces. The difference for temperature distribution can be referred in Figure 4. A significant difference can be observed in which the maximum temperature drops with increasing concentration of water vapor. Increasing water vapor concentration concurrently reduces the nitrogen content. A study analyzed the dilution effect between water vapor and nitrogen towards NOx emission of a natural gas flame. They found that at constant adiabatic temperature $T_{a d}$, the reducing effect of water vapor dilution is larger than that of nitrogen which not only reduces the NOx emission but also lowers flame temperature. The resulting trend is evident in Figure 4. Addition of water vapor apparently leads to cooling and smothering effect on the flame where dilution of the fuel and oxidizer occurs. The simulation result is supported by a study done by Ferguson and co-workers [10] who revealed that the flame temperature of an opposed diffusion flame steadily decreases with increasing water vapor mole fraction. However, up to certain mole fraction, the flame may extinguish and the associated temperature profile remains flat indicating that the flame cannot be sustained.

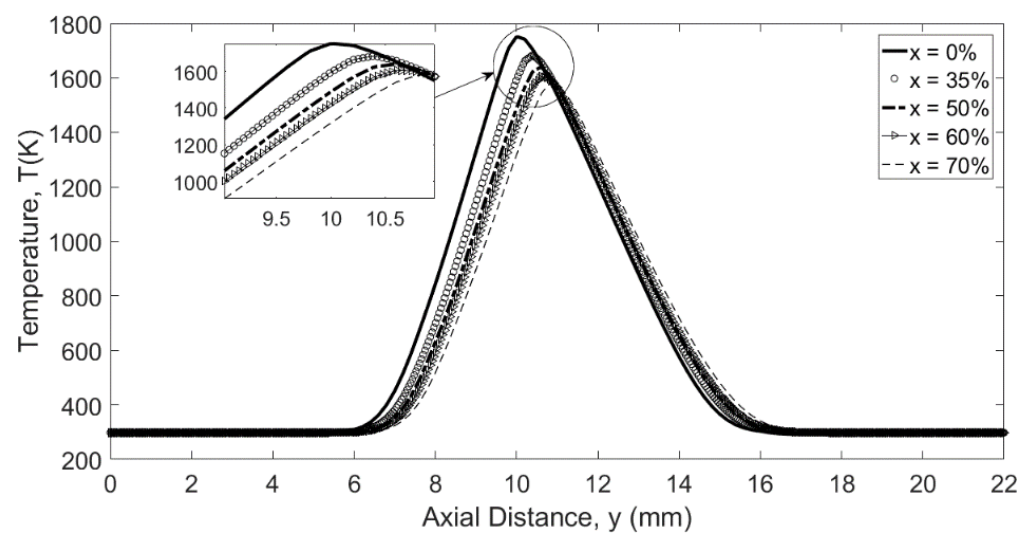

Fig. 4. Temperature distribution for different water vapor concentration

In total, there are five cases for which CNT growth at different water vapor concentrations have been simulated. As shown in Figure 5, the length profile is similar to one another where optimum growth region is found at the location of high temperature. Based on the growth rate equation, the impingement rate increases with temperature. The increase in the impingement rate is attributed to 
a high surface density of carbon atoms, $n_{1}$, deemed essential for the growth of CNT. Hence, the length and growth rate of CNT increase with $n_{1}$.

Figure 5 illustrates the growth of CNT with $0.7 \%$ of water vapor where the maximum length is observed at $56 \mu \mathrm{m}$. In general, the CNT length decreases with vapor concentration, and water vapor at $70 \%$ recorded the nethermost peak at nearly $40 \mu \mathrm{m}$.

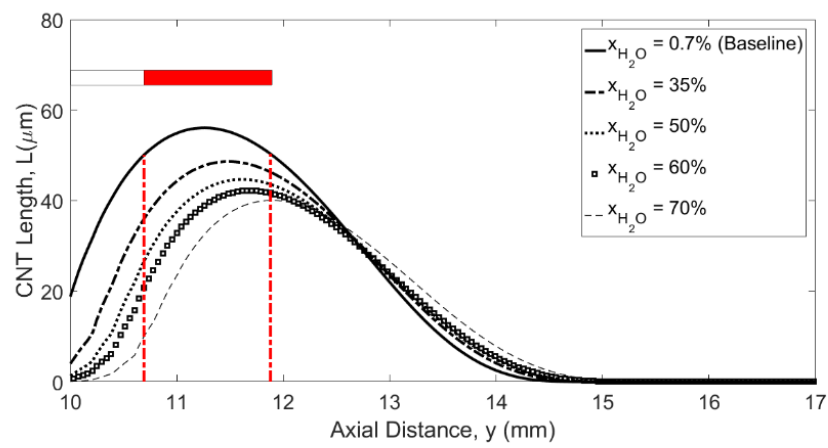

Fig. 5. CNT length for different water vapor concentration

On the other hand, the estimated CNT length for water vapor concentrations of $35 \%, 50 \%, 60 \%$ and $70 \%$ top at $48,44,42$ and $40 \mu \mathrm{m}$, respectively. This is due to the decrease in temperature that mitigate the density of carbon atom supply for the growth of CNT.

The result corresponds well with a study which reported an obvious decrease in CNT yield and length with decreasing local gas-phase temperature [11]. The rate of catalytic nanoparticle formation reduced as temperature drops. Even, no CNT was observed yielded the temperature lower than $950 \mathrm{~K}$. The inclusion of $\mathrm{C} 2 \mathrm{H} 2$ in the present study may have prolonged the catalyst lifetime. Table 1 displays the comparison for CNT growth for different water vapor concentration.

Table 1

Effect of water vapor concentration towards growth of CNT

\begin{tabular}{|c|c|c|c|}
\hline Water Vapor Concentration (\%) & $\begin{array}{c}\text { Maximum CNT Length, } \\
\boldsymbol{L}_{\max }(\boldsymbol{\mu} \mathrm{m})\end{array}$ & $\begin{array}{c}\text { Width of Optimum } \\
\text { Growth Region (mm) }\end{array}$ & $\begin{array}{c}\text { Shifting Distance } \\
\text { from Origin (mm) }\end{array}$ \\
\hline 0.7 & 56.0722 & 1.188 & 0.00 \\
\hline 35 & $48.6402(-13.1 \%)$ & $1.100(-7.4 \%)$ & $0.22(-2.0 \%)$ \\
\hline 50 & $44.6688(-20.2 \%)$ & $1.056(-11.1 \%)$ & $0.40(-3.5 \%)$ \\
\hline 60 & $42.2114(-24.6 \%)$ & $1.012(-14.8 \%)$ & $0.44(-4.0 \%)$ \\
\hline 70 & $40.0674(-28.4 \%)$ & $0.968(-18.5 \%)$ & $0.64(-5.7 \%)$ \\
\hline
\end{tabular}

\section{Conclusion}

In the present study, the sensitivity of the growth rate model employed and the influence of different catalyst use on the growth of CNTs were investigated. Fe has yielded better CNT length as compared to Co catalyst. The length of CNT utilizing Fe reached $147 \mu \mathrm{m}$ which is nearly 3 times higher than that of Co of only $56 \mu \mathrm{m}$. Water content of $100 \mathrm{sccm}$ from previous study via CVD method was chosen for the baseline case. This value was converted into concentration of $0.7 \%$, which yields new velocity input of $0.1512 \mathrm{~m} / \mathrm{s}$. The baseline model was able to reproduce the same profile where the temperature distribution as well as the growth region of CNT correspond with the experiment of non-water assisted synthesis. Variation of water vapor concentrations of $35 \%, 50 \%, 60 \%$ and $70 \%$ was compared with the baseline model. A discernible drop of peak temperature is evident with 
increasing water vapor due to the flame suppressing effect introduced by the water vapor. The study also indicates that the dilution effect of water vapor certainly stronger than nitrogen as presented by the temperature distribution for $70 \%$ of water vapor.

\section{References}

[1] lijima, Sumio. "Carbon nanotubes: past, present, and future." Physica B: Condensed Matter 323, no. 1-4 (2002): 15. https://doi.org/10.1016/S0921-4526(02)00869-4

[2] Hu, J. L., C. C. Yang, and J. H. Huang. "Vertically-aligned carbon nanotubes prepared by water-assisted chemical vapor deposition." Diamond and related materials 17, no. 12 (2008): 2084-2088. https://doi.org/10.1016/i.diamond.2008.07.010

[3] Liu, Hao, Yong Zhang, Ruying Li, Xueliang Sun, Fengping Wang, Zhifeng Ding, Philippe Mérel, and Sylvain Desilets. "Aligned synthesis of multi-walled carbon nanotubes with high purity by aerosol assisted chemical vapor deposition: Effect of water vapor." Applied surface science 256, no. 14 (2010): 4692-4696. https://doi.org/10.1016/j.apsusc.2010.02.074

[4] Zainal, Muhammad Thalhah, Mohd Fairus Mohd Yasin, and Mazlan Abdul Wahid. "Optimizing flame synthesis of carbon nanotubes: experimental and modelling perspectives." Jurnal Teknologi 78, no. 8-4 (2016). https://doi.org/10.11113/it.v78.9595

[5] Zainal, Muhammad Thalhah, Mohd Fairus Mohd Yasin, and Mazlan Abdul Wahid. "Optimizing flame synthesis of carbon nanotubes: experimental and modelling perspectives." Jurnal Teknologi 78, no. 8-4 (2016). https://doi.org/10.11113/it.v78.9595

[6] Bower, Chris, Otto Zhou, Wei Zhu, D. J. Werder, and Sungho Jin. "Nucleation and growth of carbon nanotubes by microwave plasma chemical vapor deposition." Applied Physics Letters 77, no. 17 (2000): 2767-2769.

https://doi.org/10.1063/1.1319529

[7] Lee, Cheol Jin, Jeunghee Park, and A. Yu Jeong. "Catalyst effect on carbon nanotubes synthesized by thermal chemical vapor deposition." Chemical Physics Letters 360, no. 3-4 (2002): 250-255. https://doi.org/10.1016/S0009-2614(02)00831-X

[8] Suriani, A. B., N. A. Asli, M. Salina, M. H. Mamat, A. A. Aziz, A. N. Falina, M. Maryam et al. "Effect of iron and cobalt catalysts on the growth of carbon nanotubes from palm oil precursor." In IOP Conference Series: Materials Science and Engineering, vol. 46, no. 1, p. 012014. IOP Publishing, 2013.

https://doi.org/10.1088/1757-899X/46/1/012014

[9] Hou, Shuhn-Shyurng, De-Hua Chung, and Ta-Hui Lin. "High-yield synthesis of carbon nano-onions in counterflow diffusion flames." Carbon 47, no. 4 (2009): 938-947. https://doi.org/10.1016/i.carbon.2008.11.054

[10] Ferguson, Selina C., Ambarish Dahale, Babak Shotorban, Shankar Mahalingam, and David R. Weise. "The role of moisture on combustion of pyrolysis gases in wildland fires." Combustion science and technology 185, no. 3 (2013): 435-453.

https://doi.org/10.1080/00102202.2012.726666 